\section{Refluxo laringofaringeano: estudo prospectivo correlacionando achados laringoscópicos precoces com a phmanometria de 24 horas de 2 canais}

\author{
O. Marambaia',N. A. Andrade?, \\ D.G. Varela ${ }^{3}$, M.C. Juncal ${ }^{3}$
}

Laringopharingeal reflux: prospective study that compare early laryngoscopic finds and 2 channel and 24 hours esophageal testing

Palavras-chave: refluxo, pHmetria, laringoscopia, laringite, disfonia, tosse.

Key words: reflux, pHmetry, laryngoscopy, laringitis, hoarseness, cough.

\section{I}

ntrodução: Manifestações laríngeas do refluxo gastroesofágico são problemas cada vez mais comuns. Estudos revelam alta associação com sensação de "globus", rouquidão crônica e com tosse crônica. Seu diagnóstico e tratamento diferem da clássica doença do refluxo gastro-esofágico. Os achados à endoscopia laríngea de hiperemia e edema de estruturas glóticas, espessamento do espaço interaritenóideo, granulomas, pólipos, edema de Reinke, estenose subglótica sugerem uma investigação diagnóstica completa através da pHmanometria de 24 horas, exame de maior sensibilidade e especificidade. Objetivo: correlacionar achados clínicos e laringoscópicos precoces sugestivos de refluxo gastro-esofágico com resultados da $\mathrm{pH}$ manometria de 24 horas. Avaliar terapia medicamentosa e modificações dietéticas. Forma de estudo: Clínico prospectivo. Material e Método: pacientes adultos com queixas crônicas: tosse seca, "globus", sialorréia, disfonia, pigarro, halitose e engasgos. Foram excluídos pacientes com outras patologias de vias aéreas. Endoscopia laríngea descartava aqueles que apresentassem lesões laríngeas mais avançadas. Encaminhamento à $\mathrm{pH}$ manometria e iniciado tratamento clínico. Resultados: $83,6 \%$ apresentaram refluxo patológico. Sintomas mais freqüentes: disfonia (72,5\%), pigarro $(60,8 \%)$, tosse $(29,4 \%)$, "globus" $(23,5 \%)$ e sialorréia (19,6\%). Associação de sintomas: dois $(67,4 \%)$; três $(41,2 \%)$ e quatro $(21,5 \%)$. 49 pacientes iniciaram tratamento com omeprazol (20 mg) e dieta: $83,7 \%$ cursaram com melhora dos sintomas após 6 meses. Em 95,9\% dos pacientes tratados houve melhora laringoscópica. Conclusões: Houve uma correlação importante entre história clínica e endoscopia laríngea com achados à pHmanometria de 24 horas. Outros estudos poderão fortalecer a telescopia laríngea para o diagnóstico do refluxo laringofaríngeo e seu acompanhamento. É necessária abordagem multidisciplinar, além de um aumento do grau de suspeição do especialista.

\begin{abstract}
Summary
ntroduction: Laryngeal symptoms from the gastroesophageal reflux are increasing and being more common. Studies have shown high association with e "globus", cronic hoarseness and cronic cough. The diagnosis and treatment are different from the well known gastroesophageal reflux disease. Laryngeal endoscopy is the first exam to be done in laryngeal pacients. Laryngeal redness, thickness of space between arytenoids, granuloma, polyp, Reinke's swelling, subglottic's stenosis need a complete clinical investigation. 24 hour $\mathrm{pH}$ metry and manometry are the choice, because its sensibility and espicificity. Aim: compare early clinical and laryngeal finds of laringopharingeal reflux with 24 hours pHmetry and manometry results. Evaluate responses to clinical terapy and dietary. Study design: Clinical prospective: Material and Method: adults with those cronic complaints: dry cough, "globus", to hawk, sialorrhoea, hoarseness, halitosis and choking. Pacients with another patology from airways were excluded. The laryngoscopy was important to select only recent lesions to do 24 hour pHmetry and manometry, and after if necessary try clinical treatment. Results: $83,6 \%$ had patologic reflux. More frequent symptoms were: hoarseness $(72,5 \%)$, to hawk $(60,8 \%)$, to cough $(29,4 \%)$, "globus" $(23,5 \%)$ and sialorrhoea $(19,6 \%)$. Frequency of associated symptoms: two (67,4\%); three $(41,2 \%)$ and four $(21,5 \%) .49$ pacients have begun treatment with omeprazole and diet: $83,7 \%$ became better after 6 months. $95,9 \%$ of the treated patients had improved in laryncoscopy. Conclusions: Laryngeal endoscopy and clinical history had good correlation with 24 hour pHmetry and manometry results. Other studies would estabilsh rules to diagnosis and follow patients with laryngeal endoscopy. Working with other professionals is important to solve problems at this pathology.
\end{abstract}

\footnotetext{
${ }_{1}^{1}$ Prof. Assistente da Cadeira de ORL da Escola de Medicina e Saúde Pública / Prof. Associado da Residência de ORL da Santa Casa da Misericórdia da Bahia.

${ }^{2}$ Chefe do Serviço de Otorrinolaringologia da Santa Casa da Misericórdia da Bahia, Prof. Assistente da Cadeira de ORL da Escola de Medicina e Saúde Pública. ${ }^{3}$ O torrinolaringologista da Santa Casa da Misericórdia da Bahia.
}

Trabalho realizado pelo Serviço de O torrinolaringologia e Cabeça e Pescoço da Santa Casa da Misericórdia da Bahia e Instituto de O torrinolaringologia Otaviano Andrade (INOOA) - Hospital Santa Izabel, Salvador, Bahia, Brasil

Endereço para correspondência: Praça Almeida Couto s/n, Hospital Santa Izabel, INO OA, CEP 40050-410, Salvador Bahia, Brasil.

Tel: (0xx71)326-0006/0260, (00xx71)242-0796; Fax: (0xx71)241-2623

E-mail: inooa@terra.com.br / dgvarela@bol.com.br / omarambaia@borl.com.br

Dados preliminares apresentados no 350 Congresso Brasileiro de Otorrinolaringologia - outubro/2000 - Natal

Artigo recebido em 13 de março de 2001. Artigo aceito em 10 de outubro de 2001. 


\section{INTRODUÇÃO}

As manifestações laríngeas do refluxo gastro-esofágico são problemas cada vez mais encontrados em nosso meio $0^{13,20,27}$. Estudos recentes revelam a sua associação com 25 a $50 \%$ dos pacientes com sensação de "globus" 32 , com mais de $80 \%$ dos pacientes com queixa de rouquidão crônica ${ }^{6,17}$ e com 10 a $20 \%$ dos pacientes com tosse crônica ${ }^{15,16,21,29}$

0 refluxo laringofaringeano $0^{11,13,14}$ tem características peculiares, guarda semelhanças com o refluxo de amplitude gastro-esofágica, porém apresenta pontos diferentes desde a própria fisiopatologia até respostas ao tratamento ${ }^{1}$. Ele acontece não apenas no período noturno, mas também durante o dia na posição ereta e de forma intermitente. Nele há acometimento principal do esfíncter esofagiano superio ${ }^{24}$. Sua apresentação, via de regra, faz-se através de sintomas inespecíficos como rouquidão ${ }^{11,15}$, disfagia, sensação de "globus", sialorréia, tosse seca23, engasgos 21,24,29.

Menos da metade dos pacientes podem ap resentar queixas gástricas ou esofágicas e a endoscopia digestiva alta pode não revelar sua existência em até $50 \%$ dos $\operatorname{cas}^{15,10}$. Para a efetividade da conduta terapêutica clínica inicial, além das medidas dietéticas deve-se incluir o uso de bloqueadores de canal de prótons ${ }^{13,17}$. A resposta ao tratamento clínico devidamente conduzido dará subsídios para a decisão da necessidade ou não de intervenção cirúrgica ${ }^{25}$. A opção pela cirurgia ganha cada vez mais simpatizantes, devido aos bons resultados relatados, à crescente refratariedade ao uso de medicamentos e à necessidade de terapia por tempo prolongado $0^{12,25,26}$.

A endoscopia laríngea, associada quando possível à estroboscopia, geralmente é o primeiro exame solicitado pelo otorrinolaringologista. Ao achado de alterações como hiperemia do espaço interaritenóideo ou lesões de pregas vocais como granulomas, pólipos, Edema de Reinke, estenose subglótica, faz-se importante uma investigação diagnóstica mais completa. A pH metria de 24 horas com dois ou três canais associada à manometria é o que apresenta melhor sensibilidade e especificidade $\mathrm{e}^{5,14,31,32}$. Patologias associadas como Divertículo de Zencker e Carcinoma Laríngeo ${ }^{4,9}$ não podem ser esquecidas no diagnóstico diferencial.

0 refluxo gastro-esofágico com repercussões laringofaringeanas é uma patologia freqüente, porém ainda subdiagnosticada ${ }^{16,27}$. 0 especialista, ao deparar-se com pacientes portadores de queixas laringológicas crônicas sem melhora aos tratamentos convencionais, deve obrigatoriamente incluir dentre suas suspeitas diagnósticas o refluxo laringofaringeano.

O diagnóstico correto e precoce alertará o paciente e o médico sobre os potenciais prejuízos a médio e longo prazo $^{8,11,17,18}$ e a necessidade de tratamento seja ele clínico ou cirúrgico, tentando-se desta forma evitar novos episódios de laringite e até o surgimento de metaplasias e neoplasias do trato aéreo e digestivo $0^{3,25,26}$.

\section{OBJETIVO}

0 presente trabalho tenta correlacionar achados clínicos e endoscópicos laríngeos precoces sugestivos de refluxo laringofaríngeo com os resultados da $\mathrm{pH}$ metria associada à manometria durante 24 horas.

Avaliar respostas dos pacientes diagnosticados à terapia medicamentosa e mudança de hábitos alimentares.

\section{MATERIAL E MÉTODO}

Trata-se de um estudo prospectivo onde foram incluídos pacientes adultos (maiores de 18 anos), residentes em Salvador, triados em consultório por um único médico, entre o período de janeiro/1998 a julho/2000. Necessitavam apresentar queixas crônicas (maior que 3 meses) como tosse seca, "globus", sialorréia, disfonia, pigarro, halitose e engasgos.

Após a triagem inicial, aqueles pacientes com história, exame físico ou nasofibroscopia revelando alterações nasossinusais eram eliminados do estudo, bem como os portadores de hipotiroidismo e tabagistas. Num segundo momento os participantes do estudo foram submetidos à endoscopia laríngea. Aqueles que possuíssem achados como espessamento do espaço interaritenóideo; hiperemia e/ ou edema de aritenóides; hiperemia e/ou edema ou espessamento de pregas vocais; estase salivar em seios piriformes; hiperemia de epiglote ou hipofaringe continuariam no estudo. Outras patologias benignas da laringe, como cistos, pólipos, Edema de Reinke, granulomas e estenose subglótica, foram eliminados.

Todos os pacientes foram submetidos à $\mathrm{pH}$ manometria de dois canais por 24 horas, realizada por uma única gastroenterologista. Inicialmente, o esfíncter esofagiano inferior era localizado através de manometria esofágica computadorizada e posteriormente eram posicionados dois sensores de $\mathrm{pH}$, um $20 \mathrm{~cm}$ acima do esfíncter esofagiano inferior e outro, a $5 \mathrm{~cm}$.

Os resultados entre os achados da pH manometria de 24 horas e exame laringoscópico eram comparados e a partir de então uma decisão terapêutica conjunta adotada. Os pacientes com diagnóstico firmado de refluxo gastroesofágico iniciavam tratamento com medicação bloqueadora de canais de prótons (omeprazol) associada à mudança de hábitos alimentares. A partir de então, duas reavaliações trimestrais eram realizadas. Nelas, foram comparadas freqüência e intensidade de sintomas prévios com os atuais. Novo exame endoscópico também foi considerado como critério de melhora ou piora.

\section{RESULTADOS}

Considerou-se uma amostra inicial composta por 61 pacientes sintomáticos que atendessem aos critérios de 


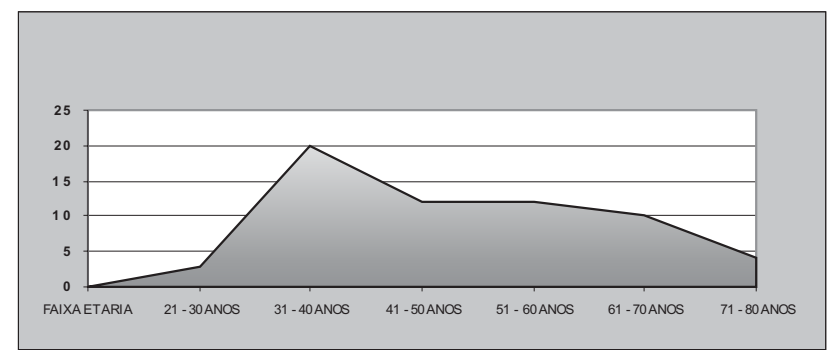

Figura 1. Distribuição de faixa etária da amostra inicial

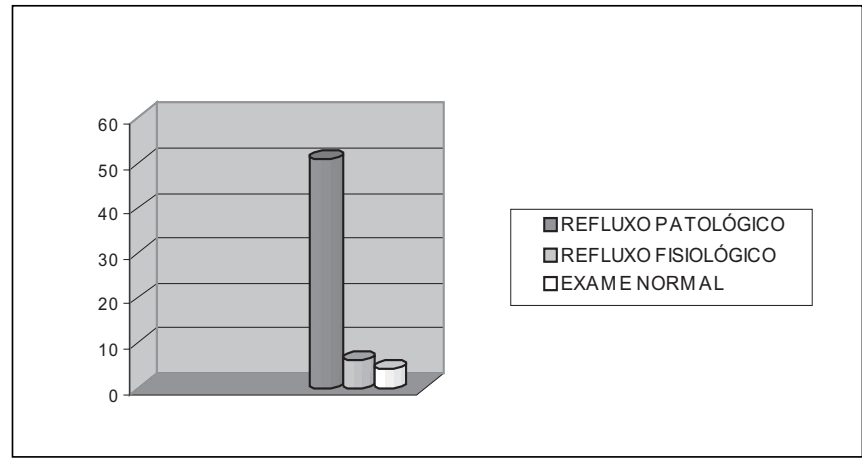

Figura 2. Resultados da phmanometria 24 horas

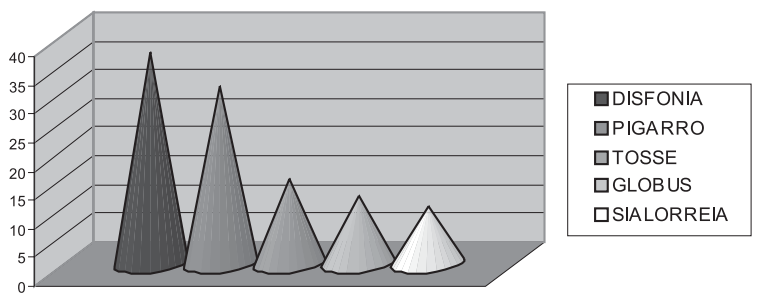

Figura 3. Distribuição dos sintomas mais comuns refluxo laringofaringeo (51 pacientes)

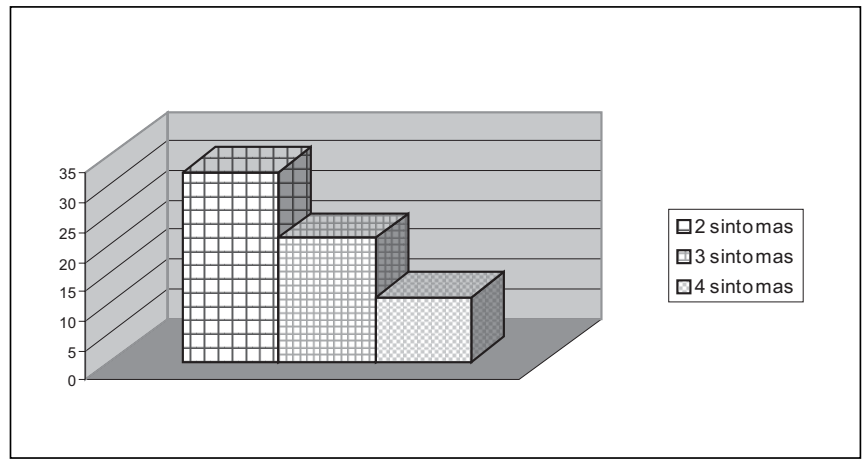

Figura 4. Distribuição do número de sintomas associados ao refluxo laringofaringeano

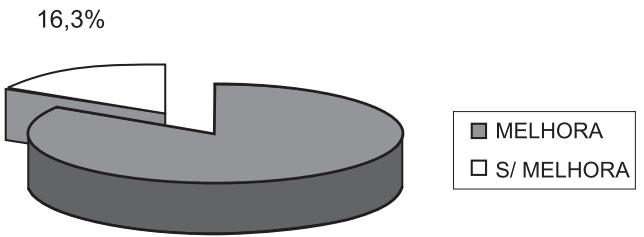

$83,7 \%$

Figura 5. Avaliação após 6 meses tratamento clínico

inclusão, tendo o mais jovem 26 anos e 0 mais velho 72 anos e sendo a média de idade de 48,44 anos. Houve um predomínio feminino de 35 (57,37\%) (Figura 1).

Cinqüenta e um pacientes $(83,6 \%)$ apresentaram exame compatível com refluxo gastro-esofágico patológico, segundo os critérios de Johnson e De Meesters, sendo que 36 deles $(73,4 \%)$ apresentavam caráter intermitente do refluxo. Um dos pacientes era portador de divertículo de Zencker (1,96\%) e outro de megaesôfago grau I (1,96\%). Seis pacientes apresentaram refluxo gastro-esofágico fisiológico $(9,8 \%), 0$ qual não levaria a sintomas laríngeos. Quatro $(6,6 \%)$ apresentaram exame dentro da normalidade (Figura 2).

Dos sintomas associados com os achados à laringoscopia, mereceram destaque a disfonia, presente em $37 \mathrm{ca}$ sos $(72,5 \%)$, o pigarro em $31(60,8 \%)$, a tosse em $15(29,4 \%)$, o "globus" em 12 (23,5\%) e a sialorréia em 10 (19,6\%). Dos 51 pacientes, $32(67,4 \%)$ apresentavam dois sintomas associados; 21 (41,2\%), três sintomas e 11 (21,5\%) compartilharam de quatro sintomas (Figura 3).

Os dois pacientes identificados como portadores de divertículo de Zencker e megaesôfago foram descartados do acompanhamento do estudo. Os 49 casos restantes iniciaram tratamento medicamentoso com bloqueador de canais de prótons (omeprazol) associado a orientações para mudança de hábitos alimentares.

Ao final da segunda avaliação trimestral, tomando-se em consideração um novo exame de endoscopia laríngea e os relatos de mudança de intensidade e freqüência dos sintomas em relação aos inicialmente coletados, houve melhora da sintomatologia em 41 pacientes $(83,7 \%)$ e manutenção ou piora em 8 pacientes (16,3\%). Em 47 pacientes (95,9\%) houve melhora endoscópica (comparação realizada por mesmo examinador) (Figura 5).

\section{DISCUSSÃO}

Os achados do presente estudo sugerem que uma anamnese bem sedimentada, aliada a achados endoscópicos laríngeos sugestivos de refluxo laringofaríngeo têm uma grande chance de correlação com alterações patológicas à 
pHmetria (83,6\%). Contudo o exame endoscópico laríngeo não conseguiu estabelecer uma gradação de gravidade do grau do refluxo, problema identificado por alguns autores em seus trabalhos ${ }^{13}$.

Devido à endoscopia digestiva alta (EDA) não ser um exame sensível para o refluxo laringofaríngeo, optou-se pela comparação direta entre a telescopia laríngea associada à anamese com a pH metria de 24 horas com dois canais e manometria, fato este corroborado pela alta percentagem de correlação encontrada (83,6\%). Na prática clínica a solicitação inicial da EDA poderá ser válida devido ao seu menor custo ${ }^{2,3,13}$.

Outro dado importante desta amostra foi o encontro de determinados sinto mas associados ao refluxo laringofaringeano: disfonia $(72,5 \%)$, pigarro $(60,8 \%)$, tosse $(29,4 \%)$, "globus" (23,5\%) e sialorréia (19,6\%). Além disso, cerca de $62,7 \%$ dos pacientes apresentavam mais de três sintomas associados, evidenciando a importância mais uma vez da anamnese na construção da suspeita clínica ${ }^{1,8,10,12}$.

0 adequado diagnóstico de refluxo gastro-esofágico com repercussões laríngeas necessita de tratamento específico ${ }^{8,19}$. De acordo com estudos recentes, a adoção de hábitos alimentares saudáveis associados ao uso de medicamentos bloqueadores do canal de prótons alcançam grande sucesso terapêutico inicial. Os pacientes do estudo apresentaram melhora sintomatológica importante (83,7\%), o que encontra-se de acordo com alguns trabalhos já publicados $\varsigma^{6,8,22,23}$, com destaque para os achados de Bauman². Os oito pacientes $(16,3 \%)$ que não responderam ao tratamento clínico inicial seriam reavaliados, novamente em três meses - nestes, o aumento na dose da medicação ou associação de outras drogas, bem como a opção cirúrgica deverão ser analisadas.

Os conhecimentos sobre refluxo laringofaríngeo encontram-se em evolução, desde o seu diagnóstico até seu tratamento. Hoje sabe-se que há alterações detectadas ao exame histológico que não são revelados à endoscopia e à pHmetria de 24 horas $^{13}$. Há autores que questionam a necessidade de exames sofisticados para a condução do refluxo gastro-esofágico e preferem o uso empírico de drogas como teste terapêutico e como ferramenta propedêutica ${ }^{7,10,12}$. A transposição destas terapêuticas para o refluxo laringofaringeo ainda não estão sedimentadas, contudo, claro que ao não se dispor de meios adequados para uma completa investigação, pode-se optar por se iniciar o tratamento ${ }^{19}$.

Estudos mais complexos com amostras maiores e de preferência com um grupo controle fornecerão resultados mais fidedignos. Contudo, é lícito supor que pacientes sem achados otorrinolaringológicos outros que as alterações laríngeas de hiperemia e/ou edema de aritenóides, epiglote e hipofaringe, espessamento do espaço interaritenóideo, ou ainda, hiperemia e/ou edema e espessamento de pregas vocais à endoscopia e que apresentem queixa de disfonia, pigarro, tosse, sensação de "globus" ou sialorréia de caráter crônico, sejam objeto de investigação mais acurada, tendo em vista a sugestiva associação entre eles e a doença do refluxo gastro-esofágico.

\section{CONCLUSÃO}

Há uma estimativa de que cerca de menos da metade dos pacientes com sintomas otorrinolaringológicos ${ }^{1,16}$ do refluxo gastro-esofágico são submetidos à correta investigação diagnóstica através da pH manometria esofágica com sensor duplo ou triplo, seja por questões econômicas ou por insuspeição.

A história clínica do paciente aliada à endoscopia laríngea, de preferência com estroboscopia, ao revelarem achados sugestivos de refluxo e sendo afastadas causa infecciosas, nasossinusais, hipotiroidismo e tabagismo merecem investigação adequada ${ }^{27}$. A endoscopia laríngea tem ganhado nos últimos anos parâmetros mais objetivos para a avaliação do grau de hiperemia, edema e lesão das estruturas glóticas e supra-glóticas. Isto torna-se importante para se tentar utilizá-la como um meio propedêutico para 0 diagnóstico inicial e acompanhamento das respostas às terapias clínicas ou cirúrgicas estabelecidas, além do controle do refluxo laringofaríngeo, mesmo quando não se dispuser da $\mathrm{pH}$ metria de 24 horas e manometria.

0 correto diagnóstico poderá trazer uma melhora na qualidade de vida do paciente e a uma prevenção de complicações ainda mais nocivas ao organismo já doente, sejam elas benignas ${ }^{17,18}$ ou malignas ${ }^{5,9}$. Daí a importância do acompanhamento multidisciplinar com gastroenterologistas, nutricionista e psicólogos.

A investigação desta patologia, que devido ao seu não-reconhecimento foi tachada várias vezes como distúrbio psicológico por alguns, pode ser hoje diagnosticada, acompanhada e bem tratada. Este estudo confirma achados da literatura internacional1,2,16,20,27,28, contudo requer ainda uma amostra maior de casos e a utilização de estudos epidemiológicos mais complexos, sejam eles populacionais ou experimentais.

\section{AGRADECIMENTOS}

Nossa gratidão à toda equipe do Serviço de Otorrinolaringologia e Cabeça e Pescoço do Hospital Santa Isabel e Instituto de O torrinolaringologia O taviano Andrade.

Nosso agradecimento especial à Dra. Helen Brum, gastroenterologista que muito nos auxiliou na realização deste estudo.

\section{REFERÊNCIASBIBLIOGRÁFICAS}

1. Ahuja V; Yencha MW; Lassen LF. Head and neck manifestatios of gastroesophageal reflux disease. Am Fam Physician, 1999 Sep, $60: 3,873-80,885-6$ 
2. Bauman NM; Bishop WP; Sandler AD; Smith RJ. Value of $p H$ probe testing in pediatric patients with extraesophageal manifestations of gastroesophageal reflux disease: a retrospective review. Ann Otol Rhinol Laryngol Suppl; 2000 Oct, 184:18-24.

3. Belafsky PC; Postman GN; Koufman JA. Laryngopharyngeal reflux symptoms improve before changes in physical findings. Laryngoscope; 2001 Jun, 111(6):979-81.

4. Biacabe B; Gleich LL; Lanccourreye O; Hartl DM; Bouchoucha M; Brasnu D. Silent gastroesophageal reflux disease in patinets wtih pharyngolaryngeal cancer further results. Head Neck, 1998 Sep, 20(6):510-4.

5. Chen RJ; Ott DJ; Casolo BJ; Moghazy KM; Koufman Ja. Correlation of laryngeal and pharyngeal carcinomas and 24-hour $\mathrm{pH}$ monitring of esophagus and pharynx. Otolaryngol Head Neck Surg, 1998 Nov, 119(5):460-2.

6. Cote DN; Miller RH. The association of gastroesophagela reflux and otolaryngologic disorders. Compr Ther, 1995, 21:2, 80-4.

7. Fass R. Empirical trials in treatment of gastroesophageal reflux disease. Dig Dis; 2000, 18(1):20-6.

8. Forrest $L A ;$ Weed $H$. Candida laryngitis appearing as leukoplakia and GERD. J. Voice; 1998 Mar, 12(1):91-5.

9. Freije JE; Beatty TW; Campbell BH; Woodson BT; Schultz CJ; Toohill RJ. Carcinoma of the larynx in patients with gastroesophageal reflux. Am J Otolaryngol; 1996 Nov-Dec, 17(6):386-90.

10. Galmiche. Treat or investigate? Eur J Gastroenterol Hepatol; 1999 Jun, 11 (Suppl 1):S11-5.

11. Gaynor EB. Laryngeal complications of GERD. J. Clin Gastroenterol; 2000 Apr, 30(3 Suppl):S31-4.

12. Gerson LB; Robbins AS; Garber A; Homberg J; Triadafilopoulos G. A cost-effectiveness analysis of prescribing strategies in the management of gastroesophagela reflux disease. Am J Gastroenterol; 2000 Feb, 95(2):395-407.

13. Haggitt RC. Department of Anatomic Pathology. Histopathology of reflux-induced esophageal and supraesophageal injuries. Am J Med; 2000 Mar 6, 108 (Suppl 4a):109S-111S.

14. Hawkins BL. Laryngopharyngeal reflux: a modern day "great masquerader". J Ky Med Assoc, 1997 Sep, 95(9):379-85.

15. Klinkeberg Knol EC. Otolaryngologic manifestation of gastroesophageal reflux disease. Scand Gastroenterol Suppl, 1998, 225, 24-8.

16. Kouffman JA: The Otolaryngologic Manifestations of Gastroesophageal Reflux Disease. Laryngoscope, 1991, 101(Supplement 53):1-78.

17. Kuhn J; Toohill RJ; Ulualp So; Kulpa J; Hofmann C; Arndorfer R Shaker R. Pharyngeal acid reflux events in patients with vocal cords nodules. Laryngoscope, 1998 Aug, 108 (8 Pt 1):1146-9.
18. Little FB, Koufman JA, Kohut RJ, et al: Effect of Gastric Acid on Pathogenesis of Subglottic stenosis. Ann O tol Rhinol Laryngol 1985, 94:516-19.

19. Metz DC; Childs ML; Ruiz C; Weinsteis GS. Pilot study of the oral omeprazole test for reflux laryngitis. Otolaryngol Head Neck Surg, 1997 Jan, 116(1):41-6.

20. Richter JE. Extraesophageal presentations of gastroesophageal reflux disease. Semin Gastrointest Dis, 1997 Apr, 8(2):75-89.

21. Richter JE; Hicks DM. Unresolved issues in gastroesophageal reflux-related ear, nose and throat problems. Am J Gatroenterol, 1997 Dec, 92(12):2143-4.

22. Ross JA; Noordzji; WooP. Voice disorders in patients with suspected laryngopharyngeal reflux disease. J. Voice, $1998 \mathrm{Mar}, 12(1): 84-8$

23. Shaw GY; Searl JP; Young JL; Miner PB. Subjective laryngoscopic and acoustic measurements of laryngeal reflux before and after treatment with omeprazole. J Voice, $1996 \mathrm{Dec}, 10(4): 410-8$.

24. Sivarao DV; Goyal RK. Functional anatomy and physiology of the upper esophageal sphincter. Am J Med, 2000 Mar, 108 (Suppl 4a):27S-37S

25. Slim K; Bousquet J; Kwiatkowski F; Lescure G; Pezet D; Chipponi J. Quality of life before and after laparoscopic fundoplication. Am J Surg; 2000 Jul, 180(1):41-5.

26. Spechler S); Lee E; Ahnen D; Goyal RK; Hirano I; Ramirez F; Raufman JP; Sampliner R; Schnell T; Sontag S; Vlahcevic ZR; Young $R ;$ Wiliford W. Long-term outcome medical and surgical therapies for gastroesophageal reflux disease: foloow-up of a randomized controlled trial. JAMA; 2001 May, 285(18):2331-8.

27. Suazo J; Facha MT; Valdovinos MA. Estudio de casos y controles de manifestaciones atipicas en la enfermedad por reflujo gastroesofagico. Case and control study of atypical manifestations in gastroesophageal reflux disease. Rev Invest Clin; 1998 JulAug, 50(4):317-22.

28. Toohill RJ; Kuhn JC. Role of refluxed acid in pathogenesis of laryngeal disorders. Am J Med, 1997 Nov, 103:5A, 100s-106s.

29. Waring JP; Lacayo L; Hunter J; Katz E; Suwak B. Chronic cough and hoarseness in patients with severe gastroesophageal reflux disease. Diagnosis and response to therapy. Dig Dis Sci, 1995 May, 40(5):1093-7.

30. Weiner GM; Batch AJ; Radford k. Dysphonia as an atypical presentation of gastroesophageal reflux. J. Laringol Otol., 1995 Dec, 109(12):1195-6.

31. Wo JM; Hunter JG; Warning JP. Dual channel ambulatory esophagela $\mathrm{pH}$ monitoring. A useful diagnostic tool? Dig Dis Sci, 1997 Nov, 42(11):2222-6.

32. Woo P; Noorzij P; Ross JA. Association of esophageal reflux and globus symptom: comparsion of laryngoscopy and 24-hour $\mathrm{pH}$ manometry. Otolaryngol head Neck Surg, $1996 \mathrm{Dec}, 115(6): 502-7$. 